\title{
Qualitative diagnostics of wind-turbine blades inspection using active thermography
}

\author{
by M. Szwedo* and P. Hellstein**
}

\author{
* MONIT SHM LLC, Lublanska 34, 31-476 Kraków, Poland, mszwedo@monitshm.pl \\ **Akademia Górniczo-Hutnicza w Krakowie, Mickiewicza 30 Ave., 30-059 Krakow, Poland, hell@agh.edu.pl
}

\begin{abstract}
Paper presents production-ready system designed for non-destructive inspection of wind-turbine blades. System is based on active thermography method with pulsed excitation. Designed and implemented by author's solution, software and image processing techniques allow implementing the system as an end line manufacturing quality control. Authors present new approach to calibrate geometrical distortion of thermal cameras with using Peltier unit matrix. Results of assessments carried out on a portion of a wind turbine blade are presented in the last part of this article.
\end{abstract}

\section{Introduction}

The application and production of composite materials in industry is rapidly growing. Most manufacturing stages are not automated and require partial, or full manual handiwork. This includes the resin impregnation and wet lay-up. Described production process may introduce variety of structural defects, such as delamination's, voids, problems associated with wet lay-up, cracks, non-uniform layer composition. These defects can become a source of future exploitation problems.

Wind energy is undergoing expansion and it is bound to grow to a commercial/consumer level in the decades to come. This growth has materialized in the form of large-scale wind farms, wind energy cooperatives, wind turbines owned by individual investors, and multinational exploration of remote sites and offshore locations. Condition monitoring and diagnostics has always been an important factor in wind energy in order to increase reliability and safety. A reliable, accurate and nondestructive damage detection method is a key element in successful monitoring of wind energy structures. A number of different damage detection methods have been developed and tested over the years including: visual inspection, passive and active approaches based on ultrasonic signals, liquid penetrant testing, eddy current based methods, radiographic methods and thermographic methods [1][2][3].

The size increase in wind power systems over the past decades lead to a greater focus on vibration problems of the structure, in particular the rotor-blades system which size and flexibility leads to high amplitude vibrations. The good balance of the rotor is important to ensure a safe, as well as an economic operation of the turbine and a good structural health preventing from early fatigue of the components, all the more so as loads are transmitted directly from the rotor to the drivetrain and generator, and can thus damage other functional subsystems.

One of the rotor imbalances type is a mass imbalance. It is the result of an inhomogeneous mass distribution across the blades caused by the manufacturing inaccuracies. This involves a greater risk of the formation of defects in the structure of the blades, which require new rapid and reliable diagnostic methods for maintaining the safety of the exploitation of wind turbines.

The monitoring and diagnostics of the blades is a complex problem, and raises several questions as on how it should be performed. The most common diagnostic method used in wind-turbine blades inspection is tap-testing. This method requires a skilled operator and gives results that are subjective. It is based on acoustic investigation. In engineering practice the success of all damage detection methods is influenced by the following major factors: (1) no need to interpret complex diagnostic data; (2) no need for baseline measurements; (3) no need for large transducer networks. There are other more advanced methods of wind-turbine blades diagnostics, but they are often complex and hard to implement in production line. The family of thermographic methods is one of the nondestructive testing techniques that meet these requirements.

The most widespread thermographic techniques utilize external heat source to deliver the required energy to a test piece. There has been a lot of research effort to develop approaches like pulsed thermography, transient thermography or lock-in thermography [4][5][6][7]. The use of thermography allows for fast, contactless, non-destructive examination of both surface and structure.

\section{System overview}

Pulsed thermography belongs to the family of active thermographic techniques with external excitation. It is schematically presented in Fig. 1. The experimental setup consists of: (1) a thermal wave source, which may be a halogen, flash or infrared lamp, (2) an infrared camera, (3) a control unit that allows to synchronize and trigger a measurement and (4) a personal computer with data processing software. If thermal source is located on the same side 
of a tested component as infrared camera, the arrangement is called the reflection mode. If it is located on the opposite side, the arrangement is called the transmission mode.

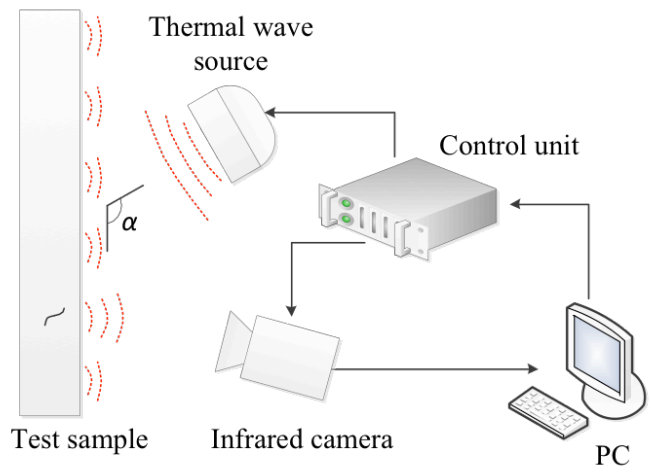

Fig. 1. Pulsed thermography - base principle of the operation

In pulsed thermography a short pulse of thermal energy is generated by a high-energy lamp and heats the surface of a tested component. Thermal waves propagate through the sample and when they come across a discontinuity the propagation path is altered, which can be observed as changes in surface temperature distribution. The pulse duration may range from several milliseconds to a few seconds. Material defects that may be present in the test sample alter the diffusion of temperature inside the sample. Diagnostic information is extracted from the anomalies in surface temperature distribution during cooling phase. One of the problems in this approach is the uniformity of delivering thermal energy to the sample. Non-uniform heating of the sample has to be compensated in the data processing phase and often results in difficulties in the extraction of damage features. Temperature field during the experiment changes at the whole surface and temperature changes between the damaged and healthy areas may be very small.

Proposed by the authors solution contains two halogen lamps that are aimed at the structure with infrared camera (FLIR A300 with wide angle lens) between them and dedicated controller. Each lamp has the power of $2 \mathrm{~kW}$. In order to precisely control power and time of excitation lamps are linked together and controlled by custom FPGA based control unit. The lamp controller could be connected wired or wirelessly using Bluetooth with heat units.

The analysis is carried out by ThermoAnalysis-WindTurbine Edition (MONIT SHM LLC) software [8] that can be run either on tablet or personal computer. It gives operator the ability to record an infrared movie, perform basic processing, modify colormap and view previously recorded files. Program is designed with simplicity in mind and can be fully operated using touchscreen monitor. It allows to quickly localise regions, which may contain defects.

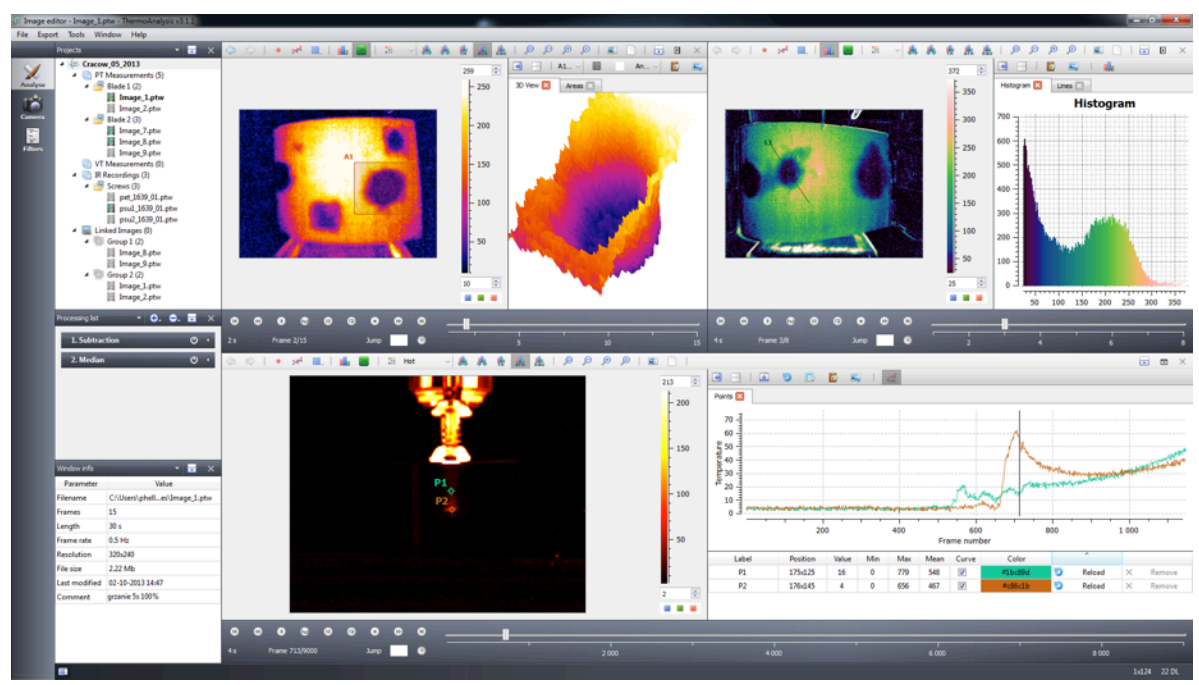

Fig. 2. ThermoAnalysis - software created by MONIT SHM LLC [8]

For more detailed analysis specialised desktop ThermoAnalysis (MONIT SHM LLC) software is provided (Fig. 2). It allows for advanced processing and analysis of thermal images to extract diagnostic features. Operator may perform geometric analysis (time history plots, area observation), create 3D representation of structure and use linear and non-linear processing functions. 


\section{Calibration}

Quality control systems are required to gather largest amount of information during one measurement cycle. In thermography it is determined by area that is covered by single IR image. This characteristic can be increased with the use of wide-angle lenses. Unfortunately lenses with large field of view introduce great amount of geometrical distortions to the image. Such images are not suitable for many vision algorithms, especially ones that deal with 3D reconstruction, pattern matching or feature extraction. In order to undistort such images, it is required to perform a spatial calibration of the camera.

\subsection{Spatial calibration overview}

Typical images produced by digital cameras are created by projecting light through set of lenses on to image sensor. This process can be described mathematically by the use of projective transformation. The projection matrix of perspective transformation may be presented in two forms - simple and full, more realistic. The first one considers only the camera and image plane positions. To fully define the perspective transformation matrix, it is crucial to know all parameters that define the camera. The process of finding true parameters of the camera that produced a given image is called camera resectioning or camera calibration. The result of this operation forms a camera model matrix, which is defined by intrinsic and extrinsic parameters. Intrinsic camera parameters characterize the optics and geometric characteristics of the camera. There are five parameters in total: focal length, principle point, skew coefficients and two distortions (radial and tangential). Extrinsic camera parameters define the camera position, characterized by its translation and rotation.

Geometric calibration is a process that has been widely discussed for normal cameras. Classical calibration setup introduced by Zhang uses a chessboard pattern to find characteristic points, which are later used to calculate the parameters forming the camera model [9]. In order to apply it to infrared camera it is necessary to heat the pattern, which has been proved to be ineffective. Vidas has proposed a modification of this method, which uses a mask-based approach [10]. Its principle is to cut out a chessboard pattern from a plate and place it in front of a heated area. This operation greatly reduces the reprojection error, because the edges of the pattern appear much sharper on thermovision image.

\subsection{Peltier unit matrix calibration}

The new proposed calibration setup modifies the mask-based method introduced by S. Vidas, by replacing the background plate with thermoelectric coolers, which work under the principle of Peltier effect. When current passes through semiconductor, heat is transferred from one side to another. This results in one side being heated, and the other one cooled. Because of that thermoelectric coolers can act both as a heater and a cooler. Peltier units can be stacked together to create Peltier modules with higher efficiency.

Peltier units allow to precisely control the temperature and keep it constant throughout calibration process. Additionally the usage of Peltier modules makes it possible to perform two types of calibration with the same setup. The first one uses the heated side of the Peltier unit, and is similar to other popular thermal cameras calibration methods. The second one uses the cold side, which can be beneficial depending on the environment and can greatly increase the contrast of observed chessboard pattern.
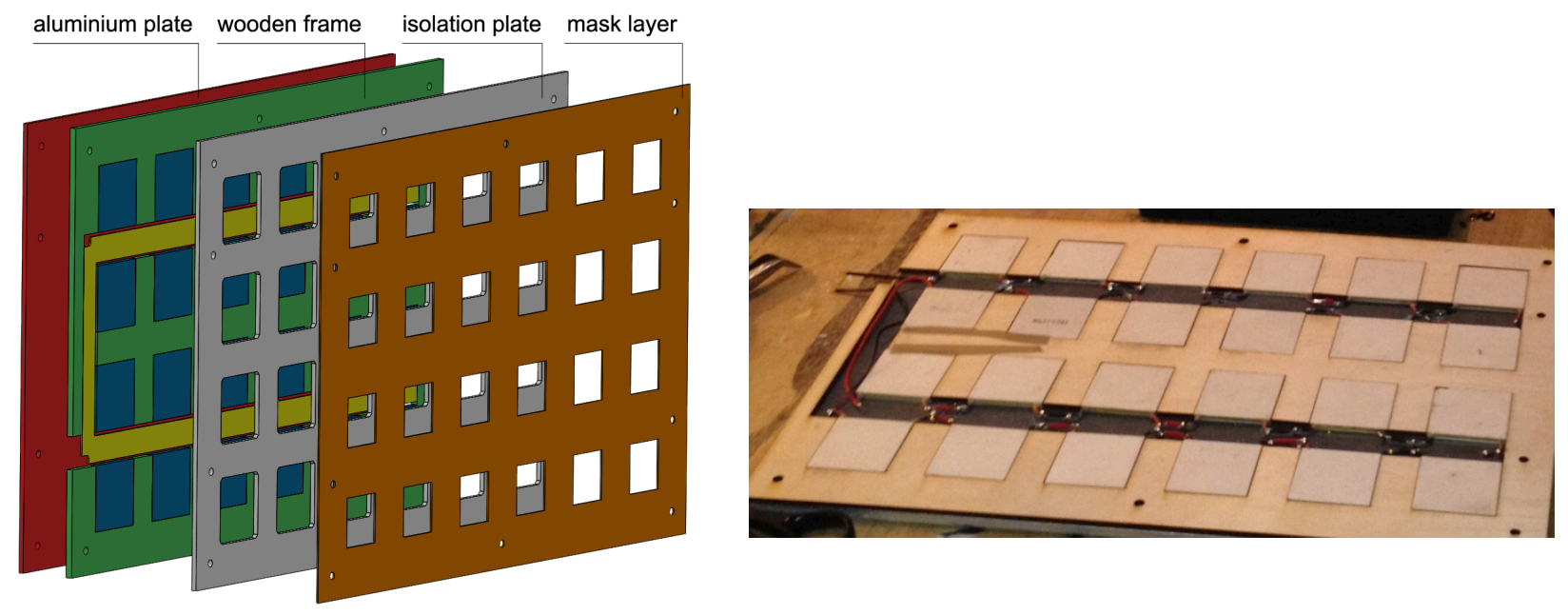

Fig. 3. Calibration pattern, exploded view design (left) and assembly photograph (right) 


\subsubsection{Calibration pattern}

The new proposed calibration pattern is made of 24 Peltiers modules (Fig. 3). This creates 96 corner points, which can be recognised by feature detection algorithm. The Peltier module that has been chosen is the TEC1-12706 model. It is a common model, which is often used for cooling processors. The maximum voltage that can be applied to it varies from 12 to $16 \mathrm{~V}$. Peltier modules are capable of producing a temperature difference of 75 degrees between the hot and cold side. The dimensions of this particular model are $40 \times 40 \times 3 \mathrm{~mm}$. The modules have been connected in series, in order to maximise the current flow, which is responsible for their effectiveness.

The calibration pattern consists of four major layers. At the back there is a $3 \mathrm{~mm}$ thick aluminum plate. It is responsible for dissipating the heat generated by the hot side of the Peltier modules. Next there is a $3 \mathrm{~mm}$ wooden frame, which holds all units. On that a $3 \mathrm{~mm}$ isolation frame and $1 \mathrm{~mm}$ mask frame are mounted. The thinner the mask frame is, the lower the calibration error will be introduced. The size of calibration squares is equal to $30 \times 30 \mathrm{~mm}$. The whole calibration pattern is screwed together and has additional handles to hold it conveniently. It is $390 \mathrm{~mm}$ wide and $270 \mathrm{~mm}$ high. It is very portable and easy to use.
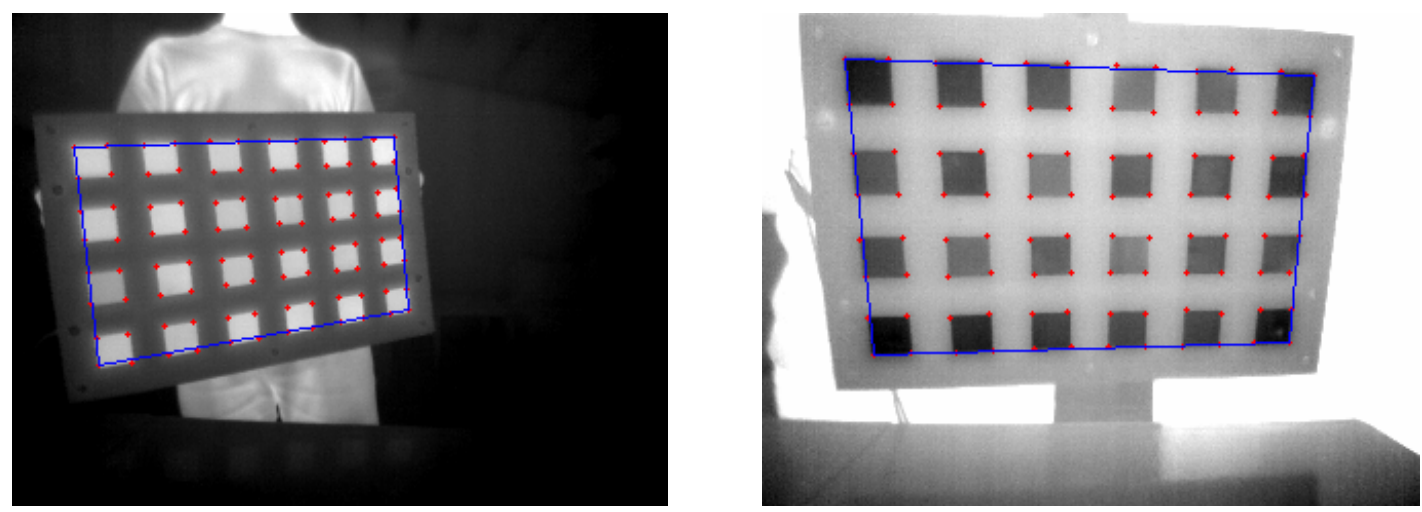

Fig. 4. Calibration pattern in two configurations

A $30 \mathrm{~W}$ power supply is attached to the calibration frame. It delivers around $1.25 \mathrm{~V}$ for each module and produces a current equal to around $0.2 \mathrm{~A}$. It is enough to create a temperature difference of about 20 degrees between the plates. The calibration pattern can be easily dismounted and the frame that holds the Peltier units can be swapped. This allows the calibration frame to create both cooled and heated chessboard pattern (Fig. 4).

\subsubsection{Software implementation}

The calibration process has been created as a module to ThermoAnalysis software [8]. It is an application designed for acquisition and analysis of infrared images. The images (or sequences of thermographic images) can be directly recorded within application using FLIR cameras. Advanced post-processing functions enable to work with single images, or a group of images at the same time. The implemented calibration algorithm can be performed both offline and online. The online method calibrates the camera when live stream from camera is present. This allows for rapid calibration and works well in not demanding environments. The offline calibration gives user much more control. Firstly the user may select, which frames should be taken into the calibration process. This allows to get rid of frames, in which the algorithm performed poorly, for example due to light reflections. Secondly the user may alter the histogram of the image, which is analysed. This operation can greatly increase the accuracy of pattern search algorithm (Fig. 5).
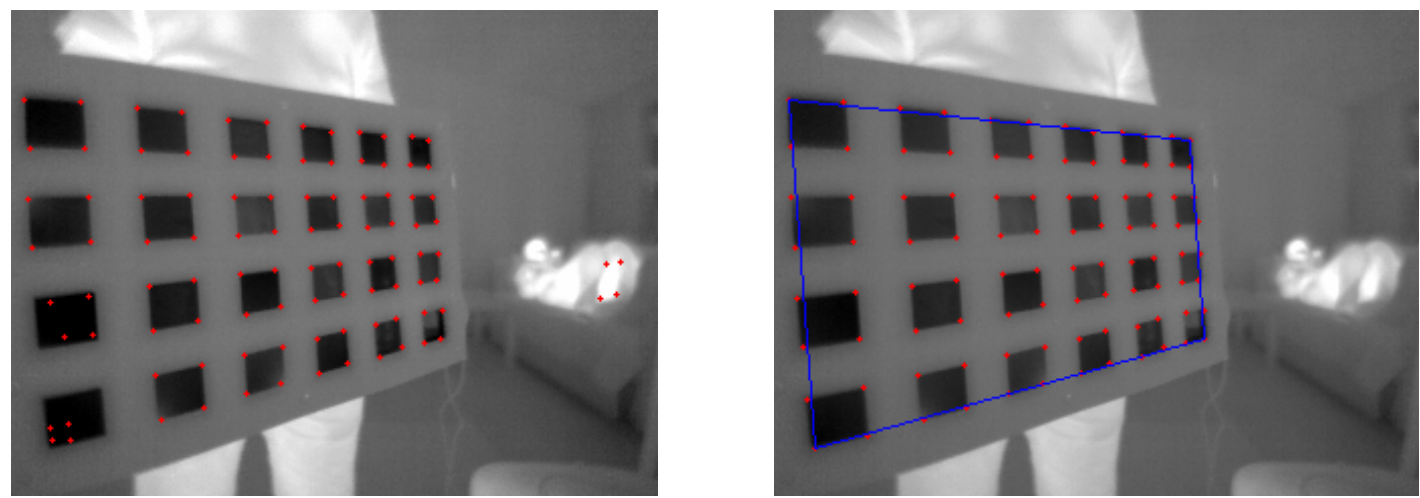

Fig. 5. Histogram optimisation in feature extraction 
The proposed calibration process makes use of the OpenCV library [11]. OpenCV has an implemention of Zhang's calibration method under the calibrateCamera() function [12]. This function takes as an input coordinates of chessboard pattern points for consecutive images. In order to obtain them, a findChessboardCorners() function has to be called. The proposed pattern differs slightly from a typical chessboard, therefore this OpenCV function had to be replaced. The new algorithm responsible for detecting chessboard corners is presented in Fig. 6. When a new frame is loaded, user may alter the histogram range of image. This operation has great impact on the result of conversion of the recorded 14 bit image to 8 bits. After that it is necessary to improve the quality of the image. The erosion and dilation operations are used to get rid of noise. Additionally an unsharp mask is applied to sharpen the edges. Next step is feature detection, which is done with Canny edge detector. The obtained edges are filtered to leave only the ones that can be approximated by a polygonal shape. The points that are left are sorted and displayed on the screen. If all 96 points where detected, information is presented to the user and points are added to the calibration stack.

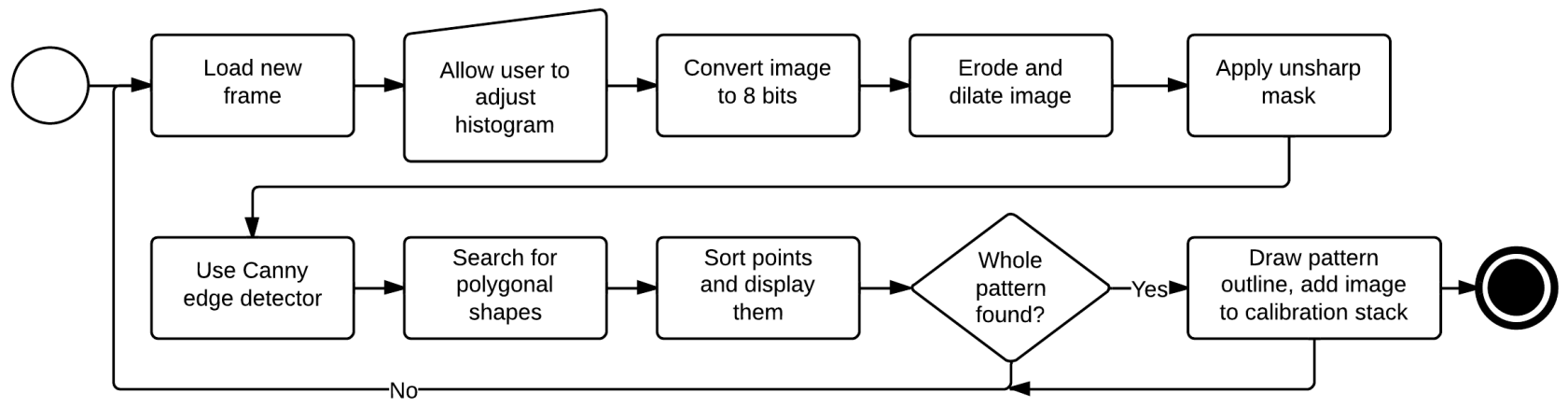

Fig. 6. Pattern detection algorithm

The minimum amount of patterns that the calibrateCamera() function can use is 8 . However it is advisable to use about 10-20 images for more accurate results. After the necessary amount of patterns has been found the camera distortion coefficients are calculated. The effectiveness of the calibration process can be evaluated by calculating the average reprojection error from all images. The calibration parameters are saved in file and can be later used to undistort the image.

\section{Results}

A series of calibration experiments has been carried out, with varying parameters and for both configurations of the calibration pattern. The thermal camera used for calibration was a FLIR A300 model, with 320x280 pixel resolution and $3 \mathrm{~Hz}$ maximum acquisition rate. The camera has been equipped with a $4 \mathrm{~mm}$ fish-eye lens, which increases its field of view from $25^{\circ} \times 18.8^{\circ}$ to $90^{\circ} \times 73^{\circ}$. The Peltier units were powered by 30 Volts. When voltage was increased, lower temperatures were achieved, which could lead to better contrast. Unfortunately those temperatures were not stable and started increasing with time. This happens because there is no heatsink or radiator mounted to the back of the Peltier modules. With greater voltage, more heat is generated and a thin aluminium plate is not enough to dissipate it. Fig. 7 presents history plots for the same point, but with different voltages applied to the calibration matrix (20,30 and 50 $\mathrm{V}$ ). It is visible that the green curve, corresponding to $50 \mathrm{~V}$, reaches the lowest temperature, but with time it gradually gets hotter. For both 20 and $30 \mathrm{~V}$ the temperature is quite stable throughout the 3-minute measurement.

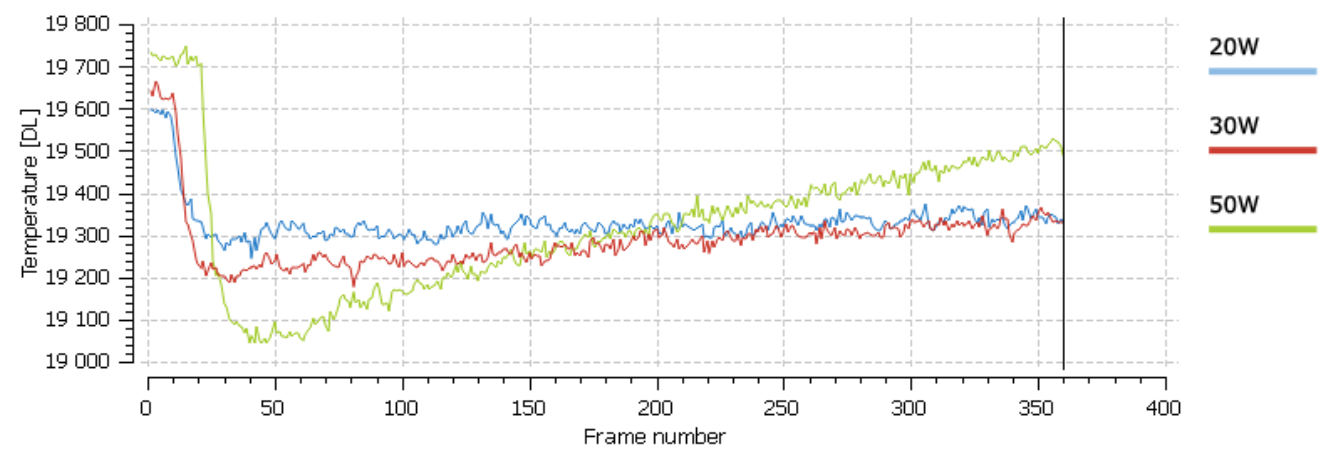

Fig. 7. Thermographs for different voltage values

Majority of Peltier modules that were used during experiments were not new. They were obtained by dismounting existing processor assemblies. Because of that their performance varies a lot. Fig. 8 presents 24 history point plots, each for one calibration square, and the corresponding thermographic image. It may be observed that the 
temperatures reached by modules are different, so is their ability to hold a steady temperature with time. Point 24 (lowest right corner of pattern) corresponds to a brand new Peltier module. It is clearly visible, that it's efficiency is the highest. Fortunately this unequeal performance of modules did not have a huge negative impact on the results of the measurements. Even with such big diversions in tempeatures the calibration algorithm proved to be robust and managed to successfully detect the chessboard pattern.
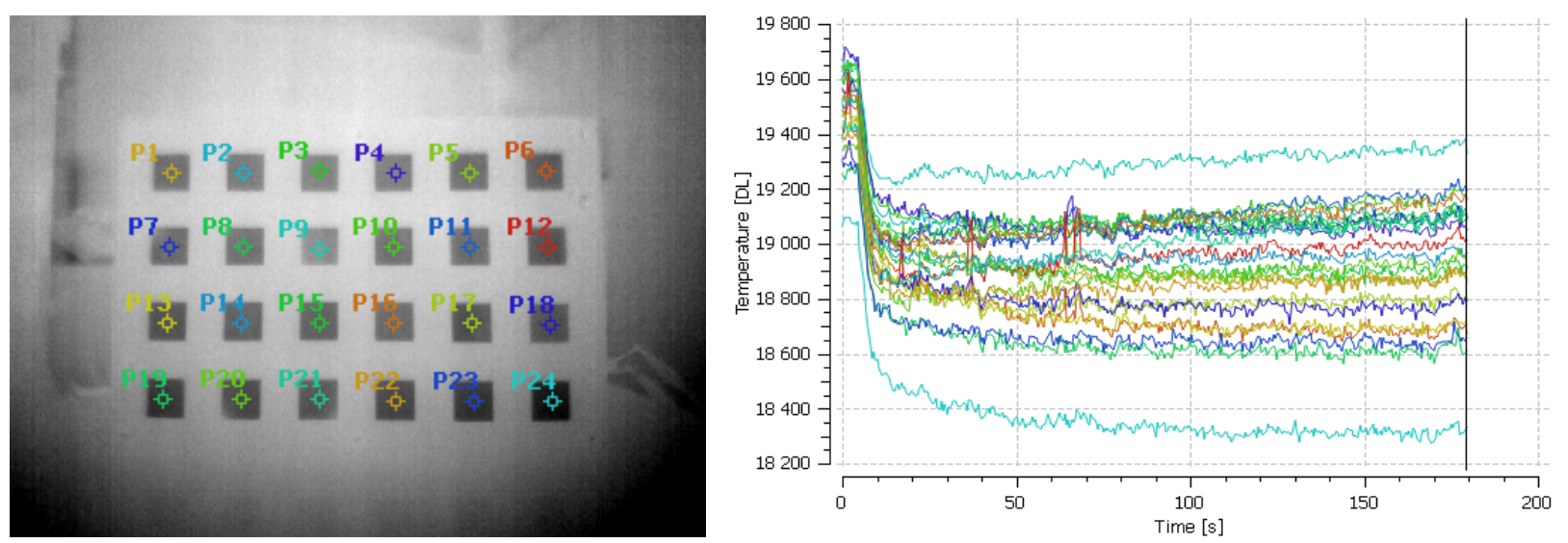

Fig. 8. Peltier modules performance comparison

The obtained calibration results are satisfactory. Fig. 9 presents an exemplary image before and after rectification. The most visible change is the reduction of radial distortions, which are the biggest problem of wide-angle lenses. The total reprojection errors that were achieved with developed calibration process were as low as 0.4 , when using the cold side configuration of the matrix. When comparing these results with commonly used heated chessboard method, it shows an improvement of about 52\% [13]. Calibration process has also been carried out with the second configuration of the calibration pattern, where the heated side of the Peltier units is exposed to the thermal camera. This setup works well with much smaller voltages. Calibration algorithm proved to be effective also in that case (Fig. 4). Both methods can be used interchangeably, depending on the ambient temperature of the room and the background environment.
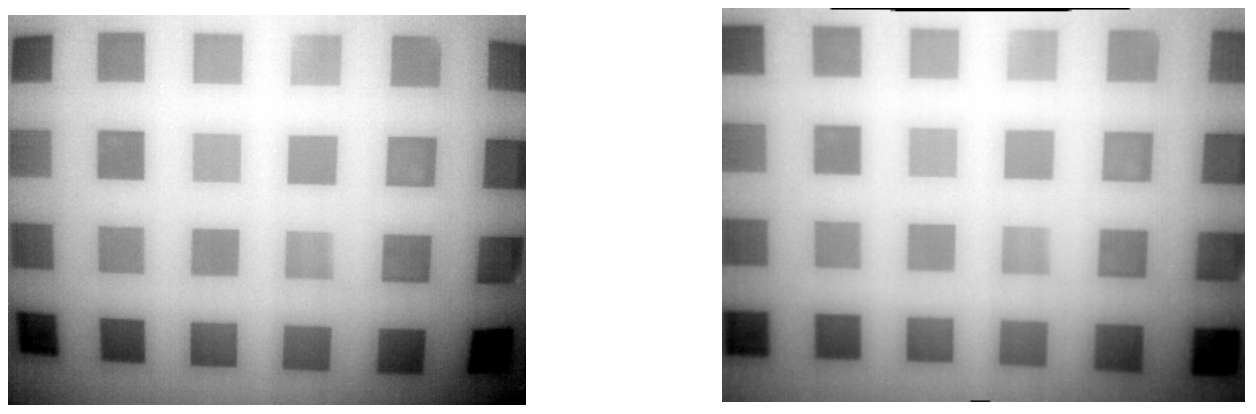

Fig. 9. Comparison of original (left) and undistorted image (right)

After calibration measurements were carried out on different wind turbine blade specimens. Initial experiments were carried out on glass fiber composite plate. During its preparation, several defects were introduced, which simulate damages of internal structure. The sample has been diagnosed with pulsed thermography method (Fig. 10).
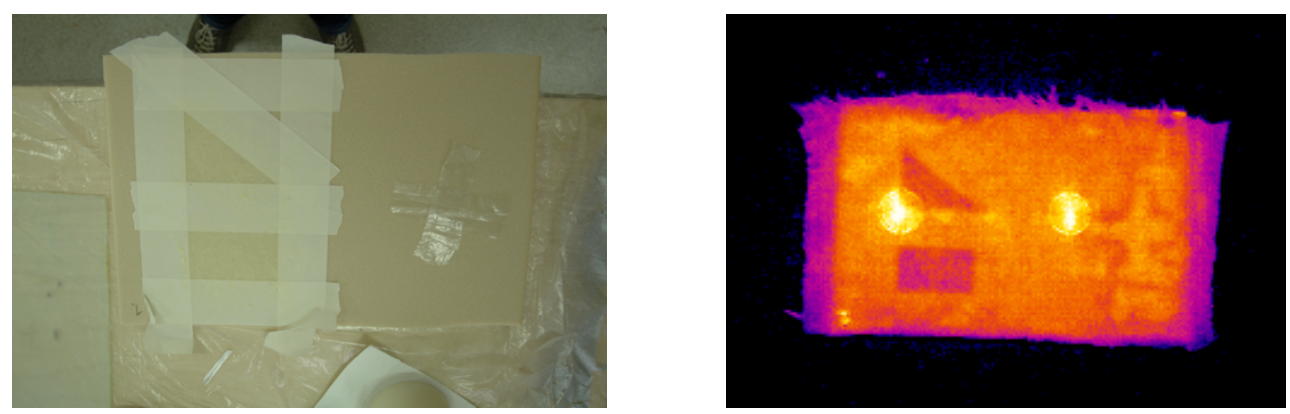

Fig. 10. Wind turbine blade specimen with identified regions 
The acquired images were later analysed in ThermoAnalysis software, with the use of Thermographic Signal Reconstruction (TSR) [14] and Pulsed Phase Thermography (PPT) [15]. Conducted experiment approved correctness of the applied measurement method and analysis techniques. Initial tests allowed to prepare final measurement system configuration, which has been later used for analysis of actual wind turbine blade.

Further analyses were performed on a prepared section of wind turbine blade with dimensions $94 \times 70 \mathrm{~cm}$. The front layer of the blade has been completely removed. After that defects were applied as if they occurred during manufacturing process. Finally the blade has been restored to its initial state using standard production methods. The surface of the blade has been heated with two high-powered $2 \mathrm{~kW}$ lamps for 10 seconds, with 40 seconds of cooling phase afterwards. Both cameras and lamp were placed $50 \mathrm{~cm}$ in front of the structure. Without the use of wide-angle lens, it would have been impossible to position them so close to the structure. Thermographic image acquired during the measurement and processed in the ThermoAnalysis software is presented in Fig. 11. Detected defects were pointed out with rectangle overlays. Areas A1, A2 and A3 present initially prepared structure inhomogeneity. Areas A4 and A5 present internal structure defects that have not been prepared during manufacturing process, but have occurred during exploitation of the blade.
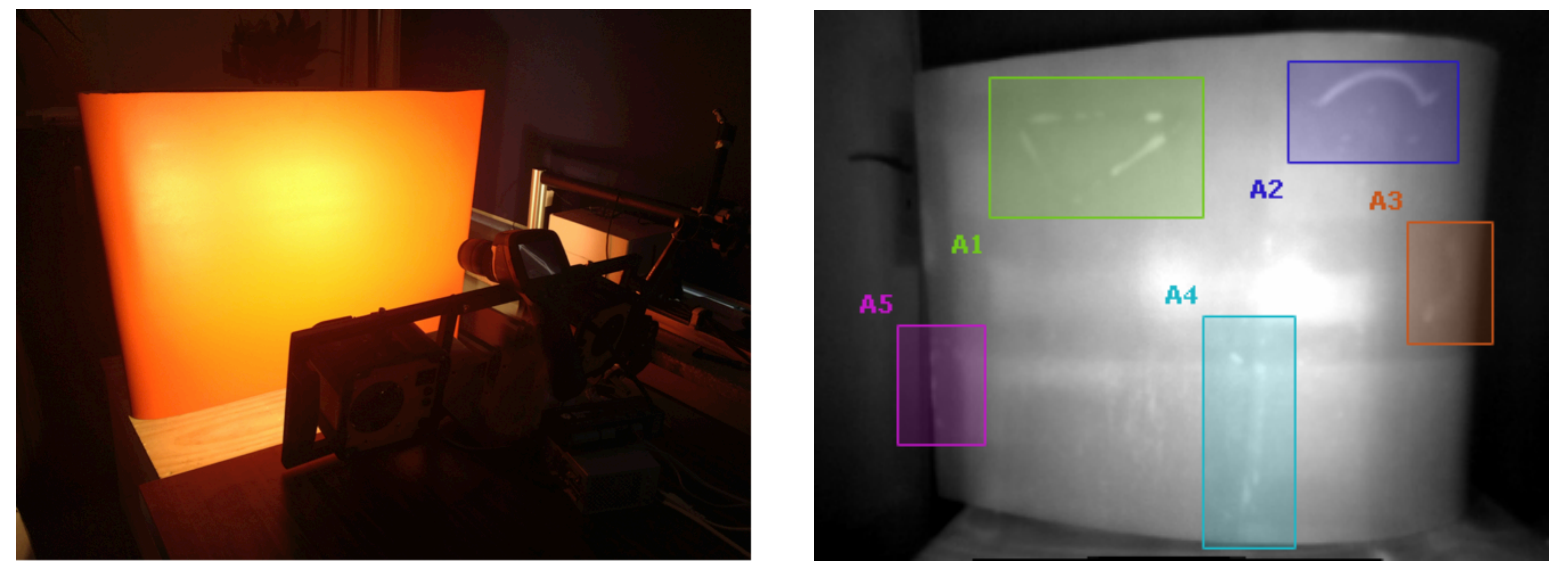

Fig. 11. Test rig with pulsed thermography measurement system (left), result of thermal image analysis with selected regions with initially prepared defects (right)

As has been described in the introduction, manufacturing of the composites initiates defects coming from the resin impregnation and wet lay-up. Described production process may introduce variety of structural defects, such as delamination's, voids, problems associated with wet lay-up, cracks, non-uniform layer composition. This problem could be rapidly identified with thermography inspection. Result of the inspection of the turbine blade with improper resin impregnation is presented in Fig. 12.
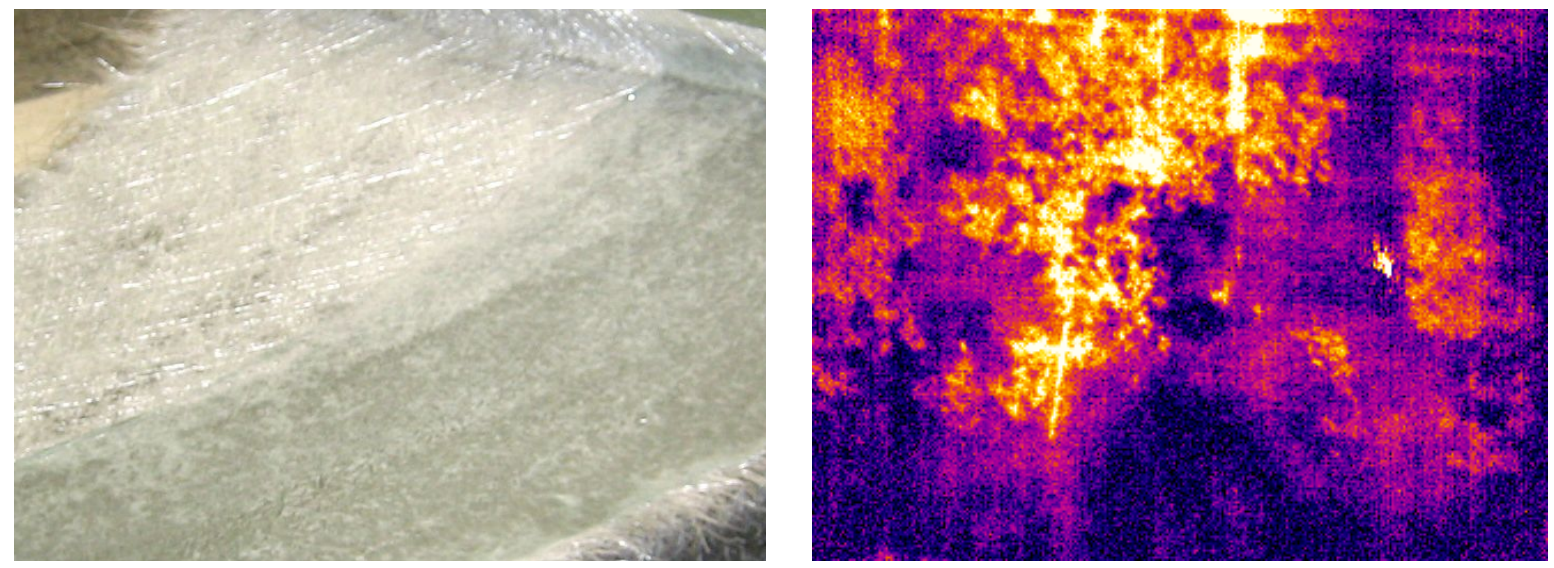

Fig. 12. Improper resin impregnation: destructive (left) and non-destructive (pulsed thermography based) investigation (right)

Acquired and processed IR images show sizes and localisation of internal defects of tested structure. Inhomogeneous thermal distribution is strictly connected with the internal state of investigated structure. The dry laminate areas are easy to identify because of its high heat capacity. These areas are visible in the IR image as brighter fields. These results allow early detection of incorrectness of blade's construction, as well as allow for their current study to rapidly obtain information about the condition of the blade or other composite based structures. 


\section{Summary}

It has been shown that it is possible to use Peltier modules for precise calibration of thermographic cameras. Geometrical calibration is a requirement, when it is necessary to enlarge the area obtained by one measurement with the use of wide-angle lenses. Such equipment introduces great radial distortions (along others), which makes it impossible to use such images in further analyses steps (such as image stitching). The usage of mask-based approach allows obtaining sharp edges, which are easily detected by image processing algorithms. Peltier modules keep constant temperature, which can be adjusted to specific needs by applying different voltages. Thermoelectric coolers can be used from both sides, hot and cold, depending on the measurement environment.

Wind-turbine blades inspections have been performed with measurement system based on pulsed thermography technique. Presented in the article results shown that proposed by the authors' solution could be adapted as a part of the diagnostic system dedicated for wind energy industry. The damage can be easily quantified with only minimal image processing. All considered measurement arrangements offer short measurement time and easy interpretation of the results, which are presented as temperature maps or three-dimensional surface plots. In case of pulsed thermography in the reflection mode, the measurements can be performed in the field, without the need to dismantle parts of the object to be tested, as only one-sided access is sufficient. Pulsed thermography as a rapidly developing NDT approach is a viable alternative to the currently used methods for the inspection of wind turbine blades.

Project co-funded by The National Centre for Research and Development.

\section{REFERENCES}

[1] Roemer J., Pieczonka L., Szwedo M, Uhl T., Staszewski W. J., "Thermography of Metallic and Composite Structures - review of applications", International Workshop on SMART MATERIALS, STRUCTURES \& SHM NDT in Canada 2013 Conference \& NDT for the Energy Industry, October 7-10, 2013 Calgary, Alberta, CANADA.

[2] W.J. Staszewski, C. Boller, G.R. Tomlinson (Eds.)., Health Monitoring of Aerospace Structures. John Wiley \& Sons, 2003.

[3] D.J. Inman, C.R. Farrar, V. Lopes Jr., V. Steffen Jr. (Eds.)., Damage Prognosis for Aerospace, Civil and Mechanical Systems, John Wiley \& Sons, 2005.

[4] J.E. Masters (Ed.)., Damage Detection in Composite Materials. Philadelphia: ASTM STP 1128 American Society for Testing and Materials, 1992.

[5] X.P.V. Maldague, Theory and practice of infrared technology for nondestructive testing, John Wiley \& Sons, 2001.

[6] O. Breitenstein, W. Warta, M. Langenkamp, Lock-in Thermography, Springer Series in Advanced Microelectronics, Springer , 2010

[7] Shepard S., 2007, Back to Basics: Thermography of Composites. ASNT Materials Evaluation, Vol.65(7), pp. 690-696.

[8] http://www.monitshm.pl/thermoanalysis, 24.04.2014.

[9] Zhang Z., "A flexible new technique for camera calibration," Pattern Analysis and Machine Intelligence, IEEE Transactions on, vol. 22, no. 11, p. 1330-1334, 2000.

[10] Vidas S., Lakemond R., Denman S., Fookes C., Sridharan S., Wark T, "A Mask-Based Approach for the Geometric Calibration of Thermal-Infrared Cameras", IEEE T. Instrumentation and Measurement 61(6): 16251635 (2012).

[11] http://opencv.willowgarage.com/wiki/, 10.04.2014.

[12] Laganière R, “OpenCV 2 Computer Vision Application Programming Cookbook", Packt Publishing, May 23, 2011.

[13] Polikovsky S., Kameda Y., Ohta Y., „Infrared and Visible Spectrum Camera Calibration”, 2011 IEICE.

[14] Shepard S.M., Ahmed T., Rubadeux B.A., Wang D., Lhota J.R., "Synthetic processing of pulsed thermographic data for inspection of turbine components", Insight, Vol. 43, No 9, Sept. 2001, British Inst. of NDT, pp. 587-589.

[15] X. Maldague, S. Marinetti, "Pulse Phase Infrared Thermography”, J. Appl. Phys., 79 (5), pp. 2694-2698, 1996. 